\title{
Efficacy of standardized training on a virtual reality simulator to advance knee and shoulder arthroscopic motor skills
}

\author{
Stefan Rahm*, Karl Wieser, David E. Bauer, Felix WA Waibel, Dominik C. Meyer, Christian Gerber \\ and Sandro F. Fucentese
}

\begin{abstract}
Background: Most studies demonstrated, that training on a virtual reality based arthroscopy simulator leads to an improvement of technical skills in orthopaedic surgery. However, how long and what kind of training is optimal for young residents is unknown. In this study we tested the efficacy of a standardized, competency based training protocol on a validated virtual reality based knee- and shoulder arthroscopy simulator.

Methods: Twenty residents and five experts in arthroscopy were included. All participants performed a test including knee -and shoulder arthroscopy tasks on a virtual reality knee- and shoulder arthroscopy simulator. The residents had to complete a competency based training program. Thereafter, the previously completed test was retaken. We evaluated the metric data of the simulator using a z-score and the Arthroscopic Surgery Skill Evaluation Tool (ASSET) to assess training effects in residents and performance levels in experts.

Results: The residents significantly improved from pre- to post training in the overall z-score: -9.82 (range, -20.35 to -1.64 ) to -2.61 (range, -6.25 to 1.5 ); $p<0.001$. The overall ASSET score improved from 55 (27 to 84) percent to 75 (48 to 92) percent; $p<0.001$. The experts, however, achieved a significantly higher $z$-score in the shoulder tasks $(p<0.001$ and a statistically insignificantly higher $z$-score in the knee tasks with a $p=0.921$. The experts mean overall ASSET score (knee and shoulder) was significantly higher in the therapeutic tasks $(p<0.001)$ compared to the residents post training result.

Conclusions: The use of a competency based simulator training with this specific device for $3-5 \mathrm{~h}$ is an effective tool to advance basic arthroscopic skills of resident in training from 0 to 5 years based on simulator measures and simulator based ASSET testing. Therefore, we conclude that this sort of training method appears useful to learn the handling of the camera, basic anatomy and the triangulation with instruments.
\end{abstract}

Keywords: Education, Simulation, Arthroscopy, Orthopaedic surgery, Virtual reality simulation, Training

\section{Background}

Training of residents is a very important, but demanding and time consuming job in teaching hospitals. Arthroscopic surgery in particular is difficult to learn since it cannot be acquired by observation and assisting alone $[1,2]$. Virtual-reality based training has become more popular in the past, but there is still a lack of a standardized integration of virtual reality based simulator training in orthopaedic post-graduate programs [3-10]. The

\footnotetext{
* Correspondence: stefan_rahm@yahoo.com

Orthopaedic Department, Balgrist University Hospital, University of Zurich Forchstrasse 340, CH-8008 Zurich, Switzerland
}

benefit of virtual-reality based teaching is proven to be at least of equal value as direct observation, animal and/ or cadaver models or videotape learning tools [11-13].

The potential $24 / 7$ availability of a training tool is increasingly important since the hands-on operation time gets less for residents. The initial high investment of such a simulator as well as the service expenses are recognized downsides. In a previous study [14] it was shown that medical students have a steep learning curve in the first 2 hours of training on a virtual reality based knee arthroscopy simulator but there were no

(c) The Author(s). 2018 Open Access This article is distributed under the terms of the Creative Commons Attribution 4.0 International License (http://creativecommons.org/licenses/by/4.0/), which permits unrestricted use, distribution, and reproduction in any medium, provided you give appropriate credit to the original author(s) and the source, provide a link to the Creative Commons license, and indicate if changes were made. The Creative Commons Public Domain Dedication waiver (http://creativecommons.org/publicdomain/zero/1.0/) applies to the data made available in this article, unless otherwise stated. 
identifiable predictors of talent or magnitude of improvement of skills.

It is not known which type of training on a virtualreality based arthroscopy simulator is the most efficient and beneficial for residents. Further, there are different types of measuring the quality of an arthroscopic procedure and there is still a lack of validated outcome score for the evaluation of an arthroscopic performance [10]. Metric data such as task time or instrument movements can be measured and the skill level of an arthroscopist can be evaluated with scores such as the Arthroscopic Surgery Skill Evaluation Tool (ASSET) $[5,15]$.

It was the purpose of this study to determine whether a standardized, competency based training advances arthroscopic skills of residents. Two hypotheses were tested: First, arthroscopic novices (residents) improve their skills significantly through a standardized competency based training program on a virtual reality simulator. Second, the residents can reach an arthroscopic proficiency skill level based on the metric data and the ASSET score compared to fellowship trained arthroscopists after the training program.

\section{Methods}

Prior to the study all participants gave their written, informed consent. Our local IRB waived the need for ethical approval; BASEC Nr: Req-2016-00442.

Twenty residents (16 males and 4 females) with a mean age of 31 (27 to 37) years and a mean of 2.5 ( 0 to 5.5 ) years experience in the orthopaedic field were included in this study. Furthermore, there were five fellowship trained experts (all males) with a mean age of 45 (34 to 63) years and a mean of 17 (8 to 35) years experience in the orthopaedic field also included in this study as a reference group. Three of them were shoulder specialists and two of them knee specialists, of which all had a very long experience in arthroscopic surgery. Detailed information on demographic data and previous experience in real and simulated arthroscopy are depicted in Table 1.

\section{Protocol}

All participants (residents and experts) performed a test on the validated VirtaMed AG (Schlieren, Switzerland) virtual reality based knee- and shoulder arthroscopy simulator $[3,16]$. The residents had to pass a competency based training program, which included several knee- and shoulder arthroscopy tasks. Thereafter, the previously completed test was redone.

\section{Test description}

To start all participants, who did not know the simulator $(n=9)$ got a standardized introduction, explaining the hard- and software. A 2 minutes hands on time trying out the test tasks was allowed to all these participants. Further, all participants could get familar with the system using the camera and the tools for exactly $60 \mathrm{~s}$.

\section{Knee}

Three knee arthroscopy tasks needed to be performed. One standard diagnostic knee arthroscopy task including the visualisation of the complete intraarticular anatomic structures. One foreign body removal task including fishing of six rings with the hook (three in the lateral and three in the medial compartment) and one guided task of removal of a flaptear of the lateral meniscus using the punch to achieve a stable meniscus. The time was stopped when the participants judged their job to be completed.

\section{Shoulder}

Twice, the same two shoulder arthroscopy tasks were performed, once in beach chair and once in lateral decubitus position. First, a standard diagnostic shoulder arthroscopy using the camera through the posterior

Table 1 Overview of the demographic information of the residents and the experts

\begin{tabular}{|c|c|c|c|c|c|c|}
\hline Total arthroscopies performed & 0 & 1 to 20 & 21 to 50 & 51 to 100 & $>100$ & $>200$ \\
\hline Residents & 6 & 1 & 5 & 0 & 0 & 0 \\
\hline Experts & 0 & 0 & 0 & 0 & 0 & 5 \\
\hline Total hours on knee / shoulder virtual reality based & 0 & 1 to 5 & 6 to 20 & & & \\
\hline Residents & 3 & 10 & 7 & & & \\
\hline Experts & 1 & 3 & 1 & & & \\
\hline Total hours of playing video games per week & 0 & 1 to 5 & 6 to 20 & & & \\
\hline Residents & 19 & 1 & 0 & & & \\
\hline Experts & 4 & 1 & 0 & & & \\
\hline Handedness & right & left & & & & \\
\hline Residents & 16 & 4 & & & & \\
\hline Experts & 5 & 0 & & & & \\
\hline
\end{tabular}

The distribution of the demographic data and previous real arthroscopy- and simulator experience are depicted of the 20 residents and the five experts 
portal visualizing the intraarticular and the subacromial (coracoacromial ligament and the acromioclavicular joint) structures was performed. Second, a therapeutic task consisting in touching five balls by the probe for 3 seconds. Either the anterior or anterolateral portal could be used.

\section{Training protocol}

The training protocol was, as mentioned before, competency based, which means, that residents with a certain level of performance regarding operation time and overall camera and instrument pathway were allowed to proceed to the next task and therefore have a shorter overall training period. Less experienced residents, which did not achieve a certain benchmark, had to repeat the tasks until it was completed in the required level. The training program was chosen to be at least 3 hours and maximum 5 hours if all tasks were repeated to the maximum. All participants began with training of knee arthroscopy. Overall 32 tasks including diagnostic and therapeutic arthroscopy were completed. The tasks comprised diagnostics, guided diagnostics, diagnostics and palpation with the probe, catching rings with the hook, foreign body removal with the grasper/hook (stars) and partial meniscectomy.

Afterwards the training continued with shoulder arthroscopy, first in beach chair and then in lateral decubitus position. Twenty tasks each including diagnostic and therapeutic arthroscopy were completed. The tasks comprised diagnostics, guided diagnostics, diagnostics and palpation with the probe, catching rings with the hook, foreign body removal with the grasp.

\section{Outcome parameters}

Metrics All the metric data (time in seconds, camera, hook/probe and punch pathway in centimeters) were recorded by the simulator system. We evaluated these metric data of the simulator itself and additionally used z-scores for comparison of the tests [14].

\section{Arthroscopic surgery skill evaluation tool (ASSET)} Furthermore, of two particular knee (one diagnostic arthroscopy/one therapeutic arthroscopy catching six rings) and two shoulder (one diagnostic arthroscopy in beach chair/one therapeutic arthroscopy palpating five balls with the probe in lateral decubitus) arthroscopy tasks, the ASSET was evaluated to measure the performance. The ASSET score is based on these following domains; safety, field of view, camera dexterity, instrument dexterity, bimanual dexterity, flow of procedure, quality of procedure, which had to be scored from one to five (one being a novice level and five an expert level). The additional domains, autonomy and added complexity of procedure were not evaluated in this study. This scoring was performed by two experienced knee and shoulder surgeons (S.R. and K.W.) in a fully blinded (pre- or post training/resident or expert) consensus read-out. As instrument and bimanual dexterity in diagnostic arthroscopies could not be evaluated, the maximum ASSET score was 25 points for the diagnostic tasks and 35 points for the therapeutic tasks, respectively.

The metric data were correlated to the ASSET score. The learning curve of the residents using the metric data and the ASSET scores were analyzed. The final post training test results of the residents were compared with the basic test results of the experts. Furthermore, the demographic data from the questionnaire were correlated with the metric data and the ASSET score of the final basic test.

\section{Statistical analysis}

Data are presented as mean and standard deviation (SD) for continuous variables and as proportion (\%) for categorical variables, if not stated otherwise. A two-tailed Kolmogorov-Smirnov test was used for testing normal distribution, if $p \leq 0.05$, the data were considered as normally distributed. The Wilcoxon signed-rank Test was used for testing differences between means of pre- to post training results for each participant. The MannWhitney U Test was used for testing differences between the expert and non-expert subpopulations.

The Pearson's Chi-squared test was applied for testing differences of distribution of categorical variables. A $p$-value of $<0.05$ was considered to be statistically significant.

For comparison of metric data of different dimensions, variables were normalized to the expert population by subtracting the individual score of each participant from the expert populations mean and dividing the difference by the expert populations standard deviation. The thereby calculated z-scores of each variable were then summed up and an arithmetic mean was calculated to obtain a single score for each task. The statistical analysis was performed by a professional bio-statistician.

\section{Results \\ Metrics}

The overall mean z-score of the basic test improved significantly pre- to post training from -9.82 (range, -20.35 to 1.64; SD 5.05 ) to -2.61 (range, -6.25 to $1.5 ; 1.63$ ); $p<0.001$. A statistically significant improvement was found in all pre- to post training metric shoulder tasks and all except two metric knee tasks. Only the hook pathway in the removal of six rings and the punch pathway in the partial meniscectomy task showed no significant difference from pre-to post training. (see Table 2). 
The experts reached an overall mean z-score of 0.0 (range, -0.55 to 0.52 ; SD 0.47 ) which was significantly better than the post training result of the residents $(p<0.001)$. The experts reached a higher mean knee $z$-score with 0.00 (range, -0.69 to 0.83 ; SD 0.61) than the post training residents result with -0.87 (range, -4.48 to 0.66 ; SD 1.50) not reaching statistical significance; $p=0.192$.

Table 2 Overview of the metric pre- to posttraining results and the expert results

\begin{tabular}{|c|c|c|c|c|c|c|c|c|c|c|c|c|c|c|c|}
\hline Test; Task & $\begin{array}{l}\text { Metric } \\
\text { Parameter }\end{array}$ & $\begin{array}{l}\text { Residents } \\
\text { Pretraining } \\
\text { Test (mean) }\end{array}$ & $\min$ & $\max$ & SD & $\begin{array}{l}\text { Residents } \\
\text { Posttraining } \\
\text { Test (mean) }\end{array}$ & $\min$ & $\max$ & SD & $\begin{array}{l}\text { Wilcoxon: } \\
\text { pre-to- } \\
\text { posttraining }\end{array}$ & $\begin{array}{l}\text { Experts } \\
\text { Test } \\
\text { (mean) }\end{array}$ & $\min$ & $\max$ & SD & $\begin{array}{l}\text { Mann-Whitney-U: } \\
\text { Residents } \\
\text { posttraining to } \\
\text { experts }\end{array}$ \\
\hline \multirow{2}{*}{$\begin{array}{l}\text { Knee: } \\
\text { Diagnostic } \\
\text { Arthroscopy }\end{array}$} & $\begin{array}{l}\text { Time } \\
(\mathrm{sec})\end{array}$ & 254 & 77 & 571 & 133 & 121 & 79 & 219 & 39 & $<0.001$ & 118 & 71 & 174 & 50 & 0.621 \\
\hline & $\begin{array}{l}\text { Camera } \\
\text { pathway } \\
(\mathrm{cm})\end{array}$ & 145 & 39 & 621 & 127 & 74 & 40 & 191 & 36 & 0.001 & 75 & 42 & 113 & 30 & 0.767 \\
\hline \multirow{3}{*}{$\begin{array}{l}\text { Knee: } \\
\text { Removal of } 6 \\
\text { rings }\end{array}$} & $\begin{array}{l}\text { Time } \\
\text { (sec) }\end{array}$ & 159 & 77 & 367 & 83 & 97 & 55 & 203 & 40 & 0.001 & 65 & 53 & 73 & 8 & 0.029 \\
\hline & $\begin{array}{l}\text { Camera } \\
\text { pathway } \\
(\mathrm{cm})\end{array}$ & 50 & 18 & 174 & 42 & 32 & 12 & 79 & 19 & 0.048 & 20 & 14 & 27 & 5 & 0.169 \\
\hline & $\begin{array}{l}\text { Hook } \\
\text { pathway } \\
(\mathrm{cm})\end{array}$ & 107 & 38 & 343 & 76 & 82 & 27 & 350 & 74 & 0.067 & 53 & 39 & 84 & 19 & 0.243 \\
\hline \multirow{3}{*}{$\begin{array}{l}\text { Knee: Lateral } \\
\text { partial } \\
\text { meniscectomy }\end{array}$} & $\begin{array}{l}\text { Time } \\
\text { (sec) }\end{array}$ & 332 & 128 & 952 & 235 & 149 & 109 & 231 & 35 & $<0.001$ & 134 & 87 & 191 & 50 & 0.408 \\
\hline & $\begin{array}{l}\text { Camera } \\
\text { pathway } \\
(\mathrm{cm})\end{array}$ & 147 & 53 & 665 & 159 & 70 & 35 & 200 & 35 & 0.001 & 87 & 47 & 135 & 35 & 0.243 \\
\hline & $\begin{array}{l}\text { Punch } \\
\text { pathway } \\
\text { (cm) }\end{array}$ & 42 & 20 & 77 & 16 & 33 & 17 & 60 & 12 & 0.057 & 46 & 22 & 77 & 25 & 0.447 \\
\hline \multirow{2}{*}{$\begin{array}{l}\text { Shoulder (BC): } \\
\text { Diagnostic } \\
\text { Arthroscopy }\end{array}$} & $\begin{array}{l}\text { Time } \\
\text { (sec) }\end{array}$ & 439 & 201 & 728 & 189 & 186 & 91 & 425 & 79 & $<0.001$ & 60 & 31 & 95 & 25 & $<0.001$ \\
\hline & $\begin{array}{l}\text { Camera } \\
\text { pathway } \\
(\mathrm{cm})\end{array}$ & 627 & 152 & 3291 & 694 & 201 & 67 & 385 & 90 & $<0.001$ & 73 & 25 & 107 & 33 & 0.001 \\
\hline \multirow{3}{*}{$\begin{array}{l}\text { Shoulder (BC): } \\
\text { Touching of } 5 \\
\text { balls }\end{array}$} & $\begin{array}{l}\text { Time } \\
\text { (sec) }\end{array}$ & 271 & 108 & 704 & 171 & 121 & 58 & 244 & 43 & $<0.001$ & 66 & 43 & 82 & 16 & $<0.001$ \\
\hline & $\begin{array}{l}\text { Camera } \\
\text { pathway } \\
(\mathrm{cm})\end{array}$ & 103 & 21 & 272 & 74 & 48 & 14 & 118 & 25 & 0.004 & 28 & 17 & 33 & 7 & 0.071 \\
\hline & $\begin{array}{l}\text { Hook } \\
\text { pathway } \\
\text { (cm) }\end{array}$ & 282 & 96 & 1018 & 233 & 101 & 55 & 286 & 54 & $<0.001$ & 59 & 33 & 80 & 21 & 0.035 \\
\hline \multirow{2}{*}{$\begin{array}{l}\text { Shoulder } \\
\text { (LAT): } \\
\text { Diagnostic } \\
\text { Arthroscopy }\end{array}$} & $\begin{array}{l}\text { Time } \\
\text { (sec) }\end{array}$ & 296 & 96 & 1026 & 213 & 151 & 77 & 399 & 72 & 0.005 & 70 & 51 & 99 & 18 & $<0.001$ \\
\hline & $\begin{array}{l}\text { Camera } \\
\text { pathway } \\
(\mathrm{cm})\end{array}$ & 288 & 79 & 1152 & 237 & 145 & 62 & 385 & 74 & 0.004 & 73 & 57 & 81 & 9 & 0.003 \\
\hline \multirow{3}{*}{$\begin{array}{l}\text { Shoulder } \\
\text { (LAT): } \\
\text { Touching of } 5 \\
\text { balls }\end{array}$} & $\begin{array}{l}\text { Time } \\
\text { (sec) }\end{array}$ & 155 & 50 & 693 & 156 & 61 & 30 & 117 & 21 & 0.003 & 42 & 31 & 57 & 11 & 0.042 \\
\hline & $\begin{array}{l}\text { Camera } \\
\text { pathway } \\
(\mathrm{cm})\end{array}$ & 93 & 20 & 444 & 116 & 38 & 16 & 66 & 15 & 0.023 & 21 & 15 & 27 & 5 & 0.015 \\
\hline & $\begin{array}{l}\text { Hook } \\
\text { pathway } \\
\text { (cm) }\end{array}$ & 182 & 53 & 505 & 122 & 72 & 27 & 144 & 36 & 0.005 & 33 & 28 & 38 & 5 & 0.003 \\
\hline
\end{tabular}


In the the experts scored a significantly higher score with 0.00 (range, -0.47 to 0.63 ; SD 0.46 ) compared to the post training $\mathrm{z}$-score of the residents -4.35 (range, -8.56 to -0.36 ; SD 2.28); $p<0.001$. The results between the lateral decubitus and the beach chair position did not significantly differ.

All except one parameter in the knee tasks (time in the knee removal of six rings) showed no significant difference between the post training result and the expert result. However, in the shoulder tasks the experts were significantly better in all but one parameter (camera pathway in beach chair position of shoulder touching five balls). A complete overview of the pre- to post training results and the expert results of every parameter is depicted in Table 2.

\section{Asset}

The mean overall ASSET score improved significantly in all four tasks from pre- to post training; from 55\% (range, 27 to 84 ) to $75 \%$ (range, 48 to 92 ); $p=0.001$. In all four tasks the residents reached a higher (or close to reference) score in the post training assessment, although the experts ASSET score was still significantly better in two tasks (knee triangulation (rings) and shoulder triangulation (balls)). In Fig. 1 the complete results of the ASSET score are summarized.

\section{Correlations}

There was a good correlation (correlation coefficient $=0.688 ; p=0.013$ ) between the overall $\mathrm{z}$-score and the overall ASSET score. There were no positive or negative correlations of the data regarding previous real or simulated experience, the handedness, the sports activity and the video games experience to the improvement of the scores or the final score.

\section{Discussion}

The most important finding in this study is that a standardized, competency based training leads to significant improvements of arthroscopic skills measured by both, metric data as well as structured assessment of performance and safety using the ASSET score. The difference between experts performance and residents post training remains significantly bigger for shoulder arthroscopy than for knee arthroscopy (see Fig. 1). Therefore, we could confirm our hypothesis that a significant improvement is achieved for residents, but had to reject the second hypothesis that a proficiency level, similar to the one of a fellowship trained arthroscopist, based on the metric data and the ASSET score can be reached by simulator training alone.

The significant improvement from pre- to post training was shown in the objective metric simulator data (overall $\mathrm{z}$-score, $p<0.001$, overall ASSET score $p<0.001)$. However, the residents improved their knee arthroscopy skills to a level almost as good as the experts, not showing a significant difference in the $\mathrm{z}$-scores; $p=0.192$. There was no correlation between the previous experience in arthroscopy and/or simulator training (especially knee arthroscopy) to the tests. On the other hand $50 \%$ of the residents already had knee arthroscopy simulator experience compared to only $30 \%$ having had shoulder. The tests in the simulated shoulder arthroscopy might have been less anatomically defined, favouring actual surgical experience and therefore explaining the somewhat smaller effect.

The clear correlation of the metric data and the ASSET score, used to measure the arthroscopic skill level including aspects such as the flow of the camera and instruments, as well as the safety aspect, such as not

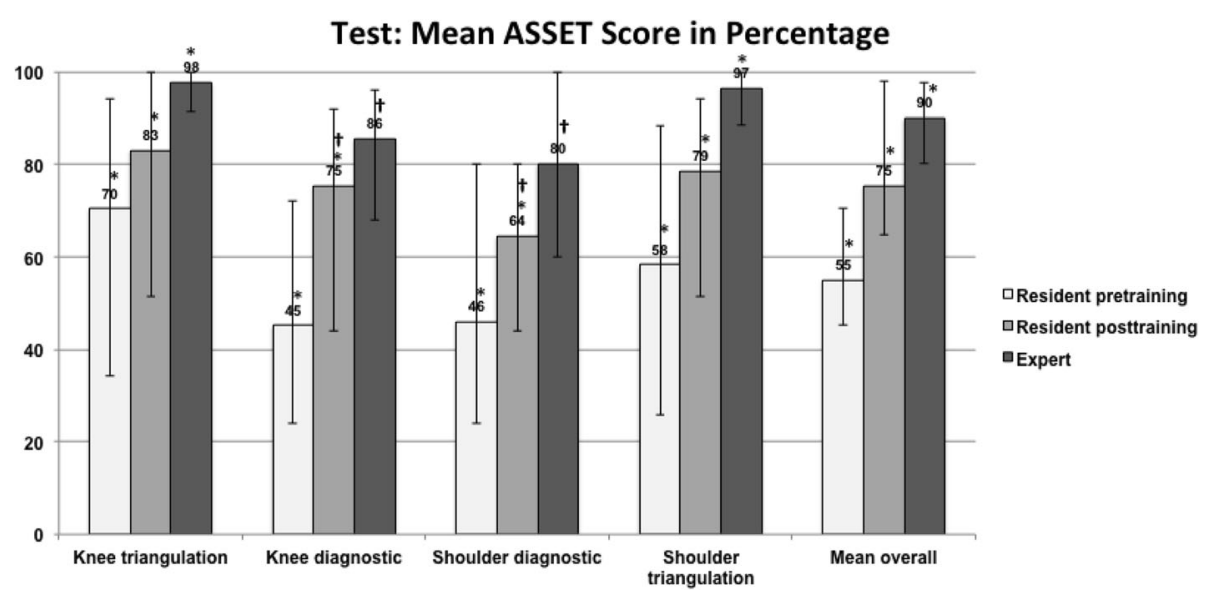

Fig. 1 Shows the percentage of the mean ASSET score of the four tested tasks. It shows a significant improvement in all four tasks from pre- to post training in the residents. Additionally there was again an improvement from the expert group with a better result in the diagnostic tasks and a significantly better result in the therapeutic tasks of knee- and shoulder arthroscopy 
scratching the intraarticular cartilage, is another important finding in this study. Good final ASSET scores of the residents in the diagnostic tasks (shoulder and knee), which were not significantly inferior to the experts, was a confirmation of our tested training program. Also, for therapeutic arthroscopy, where an additional instrument was used and triangulation required, a strong increase for the residents was seen particularly in the knee. It seems that this competency based approach works and all participants improved relevantly with a variable time, which has been spent on the simulator (fast learners vs slow learners).

We see the main value of the method in improved camera handling relative to the anatomy to be identified, as well as in the improvement of triangulation skills. For this purpose we intend to use this training program on a regular basis in the future. In addition a relevant advantage of this type of training is the independency from additional medical staff. Other practical arthroscopic issues, such as swelling of the tissue and bleeding can of course not be fully simulated with the current technology. Nevertheless, from a handling standpoint, we feel that residents with a completed simulator training have a competent skill basis for a step-wise initiation of their OR surgical training and confirm similar investigational results for the shoulder (Waterman et al. [17]) or for laparoscopic surgery (Aggarwal et al. [1]) To define a benchmark (Angelo et al. [18]) at what level real expert level is reached, remains difficult, particularly considering that the best results of the trained residents exceeded those of some experts. When looking at maximally reached values however, the best expert was usually still approximately twice as fast as the best resident, indicating an actual correlation with practical experience. Our findings are in line with a finding of Ferguson et al. who stated that basic arthroscopic skills can not immediately be transferred to an unfamiliar anatomical environment within a simulated setting [19]. In a study of Jackson et al. there is evidence, that residents can retain their skills over a time period of 6 months [20]. Our study did not test this important point in skill retaining.

As a limitation, the study group showed a natural, but large heterogeneity for the residents in all different stages with different levels and experience in real arthroscopy and on the simulator, which may explain the poor correlations with the demographic data. Still there were significant improvements found regardless the previous experience. The intensity of the training was not standardized by number of hours spend on the simulator, but we have designed a proficiency based curriculum consisting of 32 knee arthroscopy and $2 \times 20$ shoulder arthroscopy cases (to be completed in both, beach chair and lateral position). Participants had to repeat the cases until they passed a pre-defined performance level. Using this approach, we were able to have shorten the training for fast learners and for participants with higher baseline performance. On average, training time was in the range of 3-5 h (which is in accord with reports) [14]. A limitation of the current study is the fact, that the training frequency was not standardized: some of the participants completed baseline assessment, training and postassessment on 1 day. We observed that in some of these participants, the learning effects were covered by fatigue effects resulting in "negative learning effects", i.e. some participants showed lower performance after training than at baseline. As we know that more training would add some further skill improvement [21], the average training effects reported in this study are smaller than they would be in reality. Reppenhagen et al. systematically analysed optimal training frequency for arthroscopic skill acquisition and concluded that a training frequency of two sessions per week leads to best skill acquisition and retention [22].

The important question remains whether these results can be transferred in the operating room. Considering the improvement in basic arthroscopic triangulation skills, reaching for some residents the level of experts in the simulator, our results seem to support earlier findings regarding the positive transfer validity in orthopaedic surgery $[21,23]$. To lay a basis for the handling of the camera and instruments we conclude that this sort of training makes sense and should be integrated in residents training programs since the hands on time in the operating room decreases for current residents in training [24].

\section{Conclusions}

The use of programmed simulator training with this specific device for 3-5 $\mathrm{h}$ is an effective tool to advance basic motor skills of residents in training from 0 to 5 years based on simulator measures and simulator based ASSET testing. Therefore, we conclude that this sort of training method appears useful to learn the handling of the camera, basic anatomy and the triangulation with instruments.

\footnotetext{
Availability of data and materials

The datasets used and/or analysed during the current study are available from the corresponding author on reasonable request.
}

\section{Authors' contributions}

All (SR, KW, DEB, FW, DCM, CG, SFF) authors have made substantial contributions to this study; SR was involved in conception and design, analysis and interpretation of data and in drafting the manuscript. KW was involved in conception and design and interpretation of data. DB was involved in conception, analysis and statistics. FW was involved in the acquisition of data, analysis and interpretation of data. DCM, CG AND SFF were involved in conception, analysis and interpretation of data and revising the manuscript critically for important intellectual content. All authors read and approved the final manuscript. 


\section{Ethics approval and consent to participate}

Kanton Zürich, Kantonale Ethikkommission, Stampfenbachstrasse 121, 8090

Zürich, Switzerland. IRB Nr: BASEC Nr: Req-2016-00442.

All participants gave their written consent to this study.

\section{Competing interests}

The authors declare that they have no competing interests.

\section{Publisher's Note}

Springer Nature remains neutral with regard to jurisdictional claims in published maps and institutional affiliations.

Received: 17 April 2017 Accepted: 4 May 2018

Published online: 16 May 2018

\section{References}

1. Aggarwal R, Grantcharov T, Moorthy K, Hance J, Darzi A. A competencybased virtual reality training curriculum for the acquisition of laparoscopic psychomotor skill. Am J Surg. 2006;191:128-33.

2. Rosenthal R, Gantert WA, Scheidegger D, Oertli D. Can skills assessment on a virtual reality trainer predict a surgical trainee's talent in laparoscopic surgery? Surg Endosc. 2006;20:1286-90.

3. Fucentese SF, Rahm S, Wieser K, Spillmann J, Harders M, Koch PP. Evaluation of a virtual-reality-based simulator using passive haptic feedback for knee arthroscopy. Knee Surg Sports Traumatol Arthrosc. 2015:23:1077-85.

4. Haque S, Srinivasan S. A meta-analysis of the training effectiveness of virtual reality surgical simulators. IEEE Trans Inf Technol Biomed. 2006;10:51-8.

5. Koehler RJ, Nicandri GT. Using the arthroscopic surgery skill evaluation tool as a pass-fail examination. J Bone Joint Surg Am. 2013;95:e1871.

6. Modi CS, Morris G, Mukherjee R. Computer-simulation training for knee and shoulder arthroscopic surgery. ArthroscopyYJARS. Elsevier Inc. 2010;26:832-40.

7. Pedowitz RA, Marsh JL. Motor skills training in orthopaedic surgery: a paradigm shift toward a simulation-based educational curriculum. J Am Acad Orthop Surg [Internet]. 2012;20:407-9. Available from: http://eutils.ncbi. nlm.nih.gov/entrez/eutils/elink.fcgi?dbfrom=pubmed\&id= 22751159\&retmode $=$ ref\&cmd=prlinks

8. Sutherland LM, Middleton PF, Anthony A, Hamdorf J, Cregan P, Scott D, et al. Surgical Simulation. Ann Surg. 2006;243:291-300.

9. Tuijthof GJM, Visser P, Sierevelt IN, Dijk CN, Kerkhoffs GMMJ. Does perception of usefulness of arthroscopic simulators differ with levels of experience? Clin Orthop Relat Res. 2011:469:1701-8.

10. Slade Shantz JA, Leiter JRS, Gottschalk T, MacDonald PB. The internal validity of arthroscopic simulators and their effectiveness in arthroscopic education. Knee Surg Sports Traumatol Arthrosc. 2012;22:33-40.

11. Henn RF III, Shah N, Warner JJP, Gomoll AH. Shoulder arthroscopy simulator training improves shoulder arthroscopy performance in a cadaveric model. Arthroscopy. 2013:29:982-5. YJARS [Internet]. Arthroscopy Association of North America Available from: http://linkinghub.elsevier.com/retrieve/pii/ S074980631300159X

12. Basdogan C, Sedef M, Harders M, Wesarg S. VR-based simulators for training in minimally invasive surgery. IEEE Comput Graph Appl. 2007;27:54-66.

13. Moorthy K, Munz Y, Sarker SK, Darzi A. Objective assessment of technical skills in surgery. BMJ. 2003;327:1032-7.

14. Rahm S, Wieser K, Wicki I, Holenstein L, Fucentese SF, Gerber C. Performance of medical students on a virtual reality simulator for knee arthroscopy: an analysis of learning curves and predictors of performance. BMC Surg. 2016;16:14.

15. Koehler RJ, Amsdell S, Arendt EA, Bisson $\sqcup$, Bramen JP, Butler A, et al. The arthroscopic surgical skill evaluation tool (ASSET). Am J Sports Med. 2013;41: 1229-37.

16. Rahm S, Germann M, Hingsammer A, Wieser K, Gerber C. Validation of a virtual reality-based simulator for shoulder arthroscopy. Knee Surg Sports Traumatol Arthrosc. 2016;24(5):1730-7.

17. Waterman BR, Martin KD, Cameron KL, Owens BD, Belmont PJ Jr. Simulation training improves surgical proficiency and safety during diagnostic shoulder arthroscopy performed by residents. Orthopedics. 2016;39:e479-85.

18. Angelo RL, Pedowitz RA, Ryu RKN, Gallagher AG. The Bankart Performance Metrics Combined With a Shoulder Model Simulator Create a Precise and Accurate Training Tool for Measuring Surgeon Skill. YJARS. Arthroscopy Association of North America; 2015;31(9):1639-54.
19. Ferguson J, Middleton R, Alvand A, Rees J. Newly acquired arthroscopic skills: Are they transferable during simulator training of other joints? Knee Surg Sports Traumatol Arthrosc. 2017;25(2):608-15.

20. Jackson WFM, Khan T, Alvand A, Al-Ali S, Gill HS, Price AJ, et al. Learning and retaining simulated arthroscopic meniscal repair skills. J Bone Joint Surg Am [Internet]. 2012;94:e1321. Available from: http://eutils.ncbi.nlm.nih.gov/entrez/ eutils/elink.fcgi?dbfrom=pubmed\&id=22992861\&retmode=ref\&cmd=prlinks

21. Cannon WD, Garrett WE, Hunter RE, Sweeney HJ, Eckhoff DG, Nicandri GT, et al. Improving residency training in arthroscopic knee surgery with use of a virtual-reality simulator. A randomized blinded study. J Bone Joint Surg Am. 2014:96:1798-806.

22. Reppenhagen S, Zimmermann L, Buerklein D, Rudert M, Barthel T. Effectivity of arthroscopic skill acquisition in virtual reality knee arthroscopy training. Barcelona: Posterpresentation ESSKA; 2016.

23. Howells NR, Gill HS, Carr AJ, Price AJ, Rees JL. Transferring simulated arthroscopic skills to the operating theatre: a randomised blinded study. J Bone Joint Surg Br. 2008;90:494-9.

24. Aïm F, Lonjon G, Hannouche D, Nizard R. Effectiveness of Virtual Reality Training in Orthopaedic Surgery. YJARS. Arthroscopy Association of North America; 2015:32(1):224-32

\section{Ready to submit your research? Choose BMC and benefit from:}

- fast, convenient online submission

- thorough peer review by experienced researchers in your field

- rapid publication on acceptance

- support for research data, including large and complex data types

- gold Open Access which fosters wider collaboration and increased citations

- maximum visibility for your research: over $100 \mathrm{M}$ website views per year

At BMC, research is always in progress.

Learn more biomedcentral.com/submissions 